\title{
Comparison of the effectiveness of AICA-WT technique in discriminating vascular dementia EEGs
}

\begin{abstract}
The aim of the present study was to select the optimal denoising technique that helps in discriminating dementia in the early stages and illustrating its degree of severity. In this paper, a comparative analysis of three denoising techniques, which are wavelet (WT), automatic independent component analysis (AICA) rejection, and automatic hybrid technique using independent component analysis and wavelet (AICA-WT), has been conducted to select the optimal denoising technique. Two approaches have been used to extract meaningful features these are Permutation entropy (PEn) and Higuchi's fractal dimension (FD) from Electroencephalography (EEG) dataset of 5 vascular dementia (VD) patients, 15 strokerelated patients with mild cognitive impairment (MCI) and 15 healthy subjects during working memory task (WMT). k-nearest neighbors $(\mathrm{kNN})$ classifier has been used. The results show that the AICA-WT denoising technique improved the $\mathrm{kNN}$ classification accuracy from $88.15 \%$ for WT and $89.26 \%$ for AICA rejection to $90.37 \%$ for AICA-WT denoising technique. These results suggest AICA-WT consistently improves the discrimination of VD, MCI patients and control normal subjects which are useful for dementia early detection.
\end{abstract}

Keyword: Wavelet; Independent components analysis permutation entropy; Fractal dimension; K-nearest neighbors 